\title{
THE KOEBE SEMIGROUP AND A CLASS OF AVERAGING OPERATORS ON $H^{p}(D)$
}

\author{
ARISTOMENIS G. SISKAKIS
}

Abstract. We study on the Hardy space $H^{p}$ the operators $T_{F}$ given by

$$
T_{F}(f)(z)=\frac{1}{z} \int_{0}^{z} f(\zeta) \frac{1}{F(\zeta)} d \zeta
$$

where $F(z)$ is analytic on the unit disc $\mathbb{D}$ and has $\operatorname{Re} F(z) \geq 0$. Each such operator is closely related to a strongly continuous semigroup of weighted composition operators. By studying first an extremal such semigroup (the Koebe semigroup) we are able to obtain the upper bound $\left\|T_{F}\right\|_{p} \leq 2 p \operatorname{Re}(1 / F(0))+$ $|\operatorname{Im}(1 / F(0))|$ for the norm. We also show that $T_{F}$ is compact on $H^{p}$ if and only if the measure $\mu$ in the Herglotz representation of $1 / F$ is continuous.

\section{INTRODUCTION}

Let $\mathscr{P}$ be the class of functions $F(z)$ analytic on the unit disc $\mathbb{D}=\{z:|z|<$ 1\} with $\operatorname{Re} F(z) \geq 0$, and $F(z)$ is not the zero function. Let $H^{p} \quad(1 \leq p<\infty)$ be the classical Hardy spaces on $\mathbb{D}$. For $F \in \mathscr{P}$ we consider the transformation $T_{F}$ given by

$$
T_{F}(f)(z)=\frac{1}{z} \int_{0}^{z} f(\zeta) \frac{1}{F(\zeta)} d \zeta, \quad f \in H^{p} .
$$

The purpose of this paper is to study some properties of operators $T_{F}$ arising in this fashion. A well-known example of such an operator is the Cesàro operator $\mathscr{C}$. For $f(z)=\sum_{n=0}^{\infty} a_{n} z^{n}$ let

$$
\mathscr{C}(f)(z)=\sum_{n=0}^{\infty}\left(\frac{1}{n+1} \sum_{k=0}^{n} a_{k}\right) z^{n} .
$$

It is easy to see that $\mathscr{C}$ is obtained by setting $F(z)=1-z$ in (1.1). It is known [15, Theorem 1] that $\mathscr{C}$ is bounded on $H^{p}$ with norm $\|\mathscr{C}\|_{p}=p$ for $p \geq 2$ and $p \leq\|\mathscr{C}\|_{p} \leq 2$ for $1 \leq p<2$. Further, $\mathscr{C}$ is a subnormal operator on the Hilbert space $H^{2}[5,11]$.

There are however more essential reasons for studying the operators $T_{F}$; As explained in $\S 2$, these operators are closely related to a class of strongly continuous operator semigroups $\left\{S_{t}: t \geq 0\right\}$ on $H^{p}$. This was the first observed

Received by the editors June 17, 1991.

1991 Mathematics Subject Classification. Primary 47B38, 47D03, 30D55.

Part of this work was supported by a minigrant from New Mexico State University. 
in [6] for the Cesàro operator and was subsequently used in studying $\mathscr{C}$ in $[5$, 15].

The restricted growth as $|z| \rightarrow 1$ of functions in $\mathscr{P}$ translates into good behavior of the operators $T_{F}$. If $1 / F$ is bounded then $T_{F}$ is clearly a compact operator on each $H^{p}$ of norm at most $\|1 / F\|_{\infty}$. On the other hand the Cesàro operator, whose integral form contains the term $1 /(1-z)$, is not compact on any $H^{p}$ since its spectrum contains a disc [15]. The term $1 /(1-z)$ is of maximum growth for functions in $\mathscr{P}$. In this article we study some properties of $T_{F}$ in relation to the kernel $1 / F$.

The functions $F(z)$ in $\mathscr{P}$ have a Herglotz representation

$$
F(z)=\int_{\partial \mathbb{D}} \frac{\zeta+z}{\zeta-z} d \mu(\zeta)+i \gamma,
$$

where $\mu$ is a nonnegative finite Borel measure on $\partial \mathbb{D}$ and $\gamma=\operatorname{Im} F(0)$ is a real constant. We allow the zero measure $\mu=0$ to incorporate the case when $F(z)$ is an imaginary constant. In this case, however, we must have $\gamma \neq 0$. With this provision the correspondence between $F \in \mathscr{P}$ and the pairs $(\mu, \gamma)$ is unique. We say that $\mu$ has no point masses if $\mu\{w\}=0$ for each $w \in \partial \mathbb{D}$.

The main results are an upper bound for the $H^{p}$-norm of $T_{F}$ and a characterization of the compact $T_{F}$ in terms of the representing measure $\mu$ of $1 / F$. We prove the following.

Theorem 3. Suppose $1 \leq p<\infty$ and $F \in \mathscr{P}$. Then the operator $T_{F}$ is bounded on $H^{p}$ and $\left\|T_{F}\right\|_{p} \leq 2 p \operatorname{Re}(1 / F(0))+|\operatorname{Im}(1 / F(0))|$.

Theorem 4. Suppose $1 \leq p<\infty$. The operator $T_{F}$ is compact on $H^{p}$ if and only if the measure $\mu$ in the Herglotz representation of $1 / F(z)$ has no point masses.

The bound on the norm in Theorem 3 is obtained by first finding the $H^{p}$ norm of the extremal operator $K_{p}$ given in (3.4). A detailed study of a semigroup of the form (2.7) is involved (the Koebe semigroup (3.2)). As a consequence we obtain an improved bound for the norm of the Cesàro operator for $1 \leq p<3 / 2$. Also, independently of the rest of the paper, we show by a simple argument that the norm of the Cesàro operator on $H^{1}$ satisfies $\|C\|_{1}>1$ so the equation $\|\mathscr{C}\|_{p}=p$ which holds for $p \geq 2$ does not extend to $1 \leq p<2$.

For Theorem 4 we use a result of Aleman [1] and the relation of the operators $T_{F}$ to those studied in [1]. The author is indebted to A. Aleman for providing an argument leading to the proof of Theorem 4 and for other remarks on this circle of problems. Dimitri Betsakos has double-checked some of the calculations.

\section{THE OPERATORS $T_{F}$ AS RESOLVENTS}

Let $\left\{\varphi_{t}: t \geq 0\right\}$ be a semigroup, under composition, of analytic functions mapping $\mathbb{D}$ into itself, i.e.

(2.1i) $\varphi_{t}: \mathbb{D} \rightarrow \mathbb{D}$ is analytic for $t \geq 0$ and $\varphi_{0}(z)=z$.

(2.1ii) $\varphi_{t} \circ \varphi_{s}=\varphi_{t+s}$ for $t, s \geq 0$.

(2.1 iii) $\varphi_{t}(z)$ is continuous in $(t, z)$.

Such semigroups were studied in detail in [3]. If $\left\{\varphi_{t}\right\}$ is a semigroup then the functions $\varphi_{t}, t \geq 0$ have a common fixed point in the closed disc $\mathbb{D} \cup \partial \mathbb{D}$ (by a common fixed point on $\partial \mathbb{D}$ is understood a common Denjoy-Wolff point; see 
[17]). We consider here semigroups with fixed point in $\mathbb{D}$. Further, without loss of generality, we assume that the common fixed point is 0 . That is, $\left\{\varphi_{t}\right\}$ also satisfies

(2.1iv) $\varphi_{t}(0)=0$ for $t \geq 0$.

No loss of generality occurs because if the fixed point in $\mathbb{D}$ is $\neq 0$, we can compose with a Möbius automorphism of $\mathbb{D}$, and with its inverse on both sides of $\varphi_{t}$ to bring the fixed point at 0 . We denote by $\Phi$ the class of semigroups $\left\{\varphi_{t}\right\}$ satisfying $(2.1 \mathrm{i}-\mathrm{iv})$.

If $\left\{\varphi_{t}\right\} \in \Phi$ we see from [3, Theorem 3.3] that the infinitesimal generator $G(z)=\lim _{t \rightarrow 0} \partial \varphi_{t}(z) / \partial t$ of $\left\{\varphi_{t}\right\}$ has the representation $G(z)=-z F(z)$ where $F(z) \in \mathscr{P}$, and the correspondence between semigroups and the functions $F(z)$ is one-to-one. In addition, if $F(z) \in \mathscr{P}$ then there is an $\left\{\varphi_{t}\right\} \in \Phi$ having generator $-z F(z)$. Indeed if $F=c$ is constant then $\varphi_{t}(z)=e^{-c t} z$ is such a semigroup. If $F(z)$ is not constant, define

$$
h(z)=z \exp \left(\int_{0}^{z} \frac{1}{\zeta}\left(\frac{F(0)}{F(\zeta)}-1\right) d \zeta\right), \quad z \in \mathbb{D} .
$$

The function $h$ is the unique analytic solution on $\mathbb{D}$ of the initial value problem

$$
z F(z) h^{\prime}(z)=F(0) h(z), \quad h^{\prime}(0)=1 .
$$

Writing $F(0)=r e^{i \theta},-\pi / 2<\theta<\pi / 2$, we have $\operatorname{Re}\left\{e^{-i \theta} z h^{\prime}(z) / h(z)\right\}=$ $\operatorname{Re}\{r / F(z)\}>0$. Using standard facts from the theory of univalent functions [8, Theorem 2.19] we find that $h$ is one-to-one on $\mathbb{D}$ and that its range $h(\mathbb{D})$ has the following geometric property: If $w \in h(\mathbb{D})$ then the entire spiral $\{w \exp (-F(0) t): 0 \leq t<\infty\}$ joining $w$ to 0 is contained in $h(\mathbb{D})$. In the terminology of univalent function theory, $h$ is a spiral-like function (starlike if $F(0)$ is real). If we set

$$
\varphi_{t}(z)=h^{-1}\left(e^{-F(0) t} h(z)\right), \quad t \geq 0,
$$

we can easily check that $\left\{\varphi_{t}\right\} \in \Phi$ and has generator $-z F(z)$. Thus the correspondence referred to above between $\Phi$ and $\mathscr{P}$ is also onto. The function $h$ in (2.4) will be called the univalent function associated with $\left\{\varphi_{t}\right\}$. It is unique under the normalization $h^{\prime}(0)=1$.

Now let $\left\{\varphi_{t}\right\} \in \Phi$ with generator $-z F(z)$. The composition operators

$$
T_{t}(f)=f \circ \varphi_{t}, \quad t \geq 0, f \in H^{p},
$$

form a strongly continuous operator semigroup on $H^{p}$ [3]. The infinitesimal generator $\mathscr{A}_{p}$ of $\left\{T_{t}\right\}$ is the first order differential operator

$$
\mathscr{A}_{p}(f)(z)=-z F(z) f^{\prime}(z) .
$$

The weighted composition operators $\left\{S_{t}: t \geq 0\right\}$,

$$
S_{t}(f)(z)=\left(\varphi_{t}(z) / z\right) f\left(\varphi_{t}(z)\right),
$$

also form a strongly continuous semigroup [17], with generator

$$
\mathscr{B}_{p}(f)(z)=-z F(z) f^{\prime}(z)-F(z) f(z) .
$$

We observe that formally, $\left(-\mathscr{B}_{p}\right)^{-1}=T_{F}$. Thus the class of operators $\left\{T_{F}, F\right.$ $\in \mathscr{P}\}$, consists exactly of the resolvents at $\lambda=0$ of the semigroups (2.7) with 
$\left\{\varphi_{t}\right\}$ ranging in $\Phi$. It is a consequence of Theorem 3 that all such resolvents are bounded.

\section{THe Koebe SEMigroup}

The Koebe function $k(z)=z /(1-z)^{2}$ maps $\mathbb{D}$ onto the slit plane $\mathbb{C}-\{r$ : $-\infty<r \leq-1 / 4\}$. We consider the semigroup $\left\{k_{t}\right\} \in \Phi$,

$$
k_{t}(z)=k^{-1}\left(e^{-t} k(z)\right), \quad t \geq 0,
$$

with corresponding generator $G(z)=-z(1-z) /(1+z)$. The induced operator semigroup

$$
Q_{t}(f)(z)=\left(k_{t}(z) / z\right) f\left(k_{t}(z)\right), \quad f \in H^{p},
$$

will be called the Koebe semigroup. $\left\{Q_{t}\right\}$ has generator on $H^{p}$

$$
\mathscr{K}_{p}(f)(z)=-z \frac{1-z}{1+z} f^{\prime}(z)-\frac{1-z}{1+z} f(z) .
$$

We will study in detail $\left\{Q_{t}\right\}$, finding the $H^{p}$-norm and the spectrum of the operator $K_{p}=R\left(0, \mathscr{K}_{p}\right)=\left(-\mathscr{K}_{p}\right)^{-1}$, the resolvent at $0 . K_{p}$ is given by

$$
K_{p}(f)(z)=\frac{1}{z} \int_{0}^{z} f(\zeta) \frac{1+\zeta}{1-\zeta} d \zeta .
$$

The extremal properties of the Koebe function are inherited in some form, so $K_{p}$ is in some sense extremal among the operators $T_{F}$.

Most of the calculations in this section will be carried out in the Hardy spaces $H^{p}\left(\pi^{+}\right)$of the half-plane $\pi^{+}=\{z: \operatorname{Re} z>0\} . H^{p}\left(\pi^{+}\right)$consists of functions $f$ analytic on $\pi^{+}$such that

$$
\|f\|_{p}=\sup _{x>0}\left(\int_{-\infty}^{\infty}|f(x+i y)|^{p} d y\right)^{1 / p}
$$

is finite. It is well known that the boundary values of functions in $H^{p}\left(\pi^{+}\right)$ exist almost everywhere on the imaginary axis and the norm is obtained by integrating the boundary function. Also the linear map

$$
V(f)(z)=\pi^{1 / p} 2^{2 / p}(1-z)^{-2 / p} f(\psi(z)), \quad \text { where } \psi(z)=(1+z) /(1-z),
$$

is an isometry from $H^{p}\left(\pi^{+}\right)$onto $H^{p}$ [10] with inverse given by

$$
V^{-1}(f)(z)=\pi^{-1 / p}(1+z)^{-2 / p} f\left(\psi^{-1}(z)\right) .
$$

We need the following lemma.

Lemma 1. Suppose $1 \leq p<\infty$. For each $a \geq 0$, the function $\varphi_{a}(z)=\sqrt{a+z^{2}}$ induces a bounded composition operator $C_{a}(f)=f \circ \varphi_{a}$ on $H^{p}\left(\pi^{+}\right)$with norm $\left\|C_{a}\right\|_{p}=1$.

Proof. Assume first $a=1$ and let $\varphi(z)=\varphi_{1}(z)=\sqrt{1+z^{2}}$. Suppose $f(z) \in$ $H^{p}\left(\pi^{+}\right)$. Factoring out any zeros of $f(z)$ we write $f(z)=B(z) g(z)$ where $B(z)$ is a Blaschke product in the right half-plane, $|B(z)| \leq 1$ for $z \in \pi^{+}$, $|B(i t)|=1$ almost everywhere, and $g(z)$ is a zero-free function in $H^{p}\left(\pi^{+}\right)$. Then $g(z)^{p} \in H^{1}\left(\pi^{+}\right)$and

$$
|f(\varphi(z))|^{p} \leq|f(\varphi(z)) / B(\varphi(z))|^{p}=|g(\varphi(z))|^{p}
$$


for each $z \in \pi^{+}$. Since $g(z)^{p} \in H^{1}\left(\pi^{+}\right)$, it can be written as a Poisson integral of its boundary values

$$
g(w)^{p}=\frac{1}{\pi} \int_{-\infty}^{\infty} g(i t)^{p} P_{t}(w) d t, \quad w \in \pi^{+},
$$

where

$$
P_{t}(w)=\operatorname{Re}\left(\frac{1}{w-i t}\right)=\frac{x}{x^{2}+(y-t)^{2}}
$$

is the Poisson kernel at $w=x+i y$. Let $w=\varphi(z)$ in (3.5), fix $x>0$, take absolute values and integrate on the line $\operatorname{Re} z=x$ to obtain

$$
\begin{aligned}
\int_{-\infty}^{\infty}|f(\varphi(x+i y))|^{p} d y & \leq \int_{-\infty}^{\infty}|g(i t)|^{p} \frac{1}{\pi} \int_{-\infty}^{\infty} P_{t}(\varphi(x+i y)) d t d y \\
& =\int_{-\infty}^{\infty}|f(i t)|^{p} \frac{1}{\pi} \int_{-\infty}^{\infty} P_{t}(\varphi(x+i y)) d y d t
\end{aligned}
$$

We wish to show that

$$
\frac{1}{\pi} \int_{-\infty}^{\infty} P_{t}(\varphi(x+i y)) d y=\frac{1}{\pi} \int_{-\infty}^{\infty} P_{t}\left(\sqrt{1+(x+i y)^{2}}\right) d y=1,
$$

independent of $x$ and $t$. For this purpose fix $t>0$ (the case $t<0$ being similar), and consider the analytic function

$$
A(z)=\frac{1}{\sqrt{1+z^{2}}-i t}-\frac{t}{\sqrt{1+t^{2}}} \frac{1}{z-i \sqrt{1+t^{2}}}, \quad z \in \pi^{+} .
$$

Set $\tau=\sqrt{1+t^{2}}$. If $r>\tau$ it is clear that $A(z)$ is bounded on $|z| \geq r$. It is also bounded on $\pi^{+} \cap\{|z|<r\}$ because we have effectively removed the singular part of $1 /\left(\sqrt{1+z^{2}}-i t\right)$ at the point $i \tau$. Thus setting $U(z)=\operatorname{Re}(A(z))$ we have

$$
U(z)=P_{t}(\varphi(z))-(t / \tau) P_{\tau}(z) .
$$

Writing the bounded harmonic function $U(z)$ as a Poisson integral of its boundary values we have from (3.10)

$$
P_{t}(\varphi(z))=\frac{1}{\pi} \int_{-\infty}^{\infty} P_{s}(z) U(i s) d s+\frac{t}{\tau} P_{\tau}(z) .
$$

Integrating both sides of (3.11) with respect to $y$ on $\operatorname{Re} z=x$, changing the order of the integration, and using the fact that $\int P_{t}(x+i y) d y=\pi$, we have

$$
\begin{aligned}
\frac{1}{\pi} \int_{-\infty}^{\infty} P_{t}(\varphi(x+i y)) d y= & \frac{1}{\pi} \int_{-\infty}^{\infty} U(i s) \frac{1}{\pi} \int_{-\infty}^{\infty} \frac{x}{x^{2}+(y-s)^{2}} d y d s \\
& +\frac{t}{\tau} \frac{1}{\pi} \int_{-\infty}^{\infty} \frac{x}{x^{2}+(y-\tau)^{2}} d y \\
= & \frac{1}{\pi} \int_{-\infty}^{\infty} U(i s) d s+\frac{t}{\tau} .
\end{aligned}
$$

The last integral in (3.12) can be found explicitly; the boundary function of $U(z)$ is

$$
U(i s)=\lim _{x \rightarrow 0} U(x+i s)= \begin{cases}\sqrt{1-s^{2}} /\left(1-s^{2}+t^{2}\right), & -1 \leq s \leq 1, \\ 0, & |s|>1, s \neq \tau .\end{cases}
$$


An integration gives

$$
\int_{-1}^{1} \frac{\sqrt{1-s^{2}}}{1-s^{2}+t^{2}} d s=\pi\left(1-\frac{t}{\sqrt{1+t^{2}}}\right)=\pi\left(1-\frac{t}{\tau}\right),
$$

so the left-hand side integral in (3.12) has the value 1 and (3.8) has been proved. From (3.7) we have

$$
\int_{-\infty}^{\infty}|f(\varphi(x+i y))|^{p} d y \leq \int_{-\infty}^{\infty}|f(i t)|^{p} d t=\|f\|_{p}^{p} .
$$

Taking the supremum on $x>0$ in (3.13) we obtain $\left\|C_{1}\right\|_{p} \leq 1$.

Now let $a>0$. We observe that $C_{a}=M_{(1 / \sqrt{a})} \circ C_{1} \circ M_{\sqrt{a}}$ where $M_{s}, s>0$, is the operator $f(z) \rightarrow f(s z)$. A change of variable in the integral giving the norm in $H^{p}\left(\pi^{+}\right)$gives $\left\|M_{s}\right\|_{p}=s^{-1 / p}$, and we find

$$
\left\|C_{a}\right\|_{p} \leq\left\|M_{(1 / \sqrt{a})}\right\|_{p}\left\|C_{1}\right\|_{p}\left\|M_{\sqrt{a}}\right\|_{p}=\left\|C_{1}\right\|_{p} \leq 1 .
$$

But $M_{s}^{-1}=M_{1 / s}$ so we also have $\left\|C_{1}\right\|_{p} \leq\left\|C_{a}\right\|_{p}$. Thus $\left\|C_{a}\right\|_{p}=\left\|C_{1}\right\|_{p}=$ $r \leq 1$ for each $a>0$. Finally observe that operators $C_{a}$ satisfy the semigroup property $C_{a} \circ C_{b}=C_{a+b}$ for $a, b>0$, from which it follows that $r$ cannot be strictly less than 1 , completing the proof.

We now return to the Koebe semigroup $\left\{Q_{t}\right\}$. The following theorem gives the norm and the spectrum of the operators $Q_{t}$ and of the corresponding resolvent $K_{p}$.

Theorem 1. Suppose $1 \leq p<\infty$. Then we have:

(i) For each $t \geq 0$ the operator $Q_{t}$ on $H^{p}$ has norm $\left\|Q_{t}\right\|_{p}=e^{-t / 2 p}$ and spectrum $\sigma\left(Q_{t}\right)=\left\{z:|z| \leq e^{-t / 2 p}\right\}$.

(ii) The infinitesimal generator $\mathscr{K}_{p}$ has spectrum $\sigma\left(\mathscr{K}_{p}\right)=\{z: \operatorname{Re} z \leq$ $-1 / 2 p\}$.

(iii) The resolvent $K_{p}=R\left(0, \mathscr{K}_{p}\right)$ has norm $\left\|K_{p}\right\|=2 p$ and spectrum $\sigma\left(K_{p}\right)=\{z:|z-2 p| \leq 2 p\}$.

Proof. We first show that $\left\|Q_{t}\right\|_{p} \leq e^{-t / 2 p}$. Using the isometries $V$ and $V^{-1}$ we set $Q_{t}^{+}=V^{-1} \circ Q_{t} \circ V$, a semigroup in $H^{p}\left(\pi^{+}\right)$with $\left\|Q_{t}^{+}\right\|_{p}=\left\|Q_{t}\right\|_{p}$. We have

$$
Q_{t}^{+}(f)(z)=e^{-t}\left(\frac{1+z}{1+\Psi_{t}(z)}\right)^{2-2 / p} f\left(\Psi_{t}(z)\right), \quad f \in H^{p}\left(\pi^{+}\right)
$$

where

$$
\Psi_{t}(z)=\psi\left(k_{t}\left(\psi^{-1}(z)\right)\right)=\sqrt{e^{-t} z^{2}+1-e^{-t}} .
$$

The computation is straightforward and we only mention here that whenever terms of the form $k_{t}\left(\psi^{-1}(z)\right)$ appear we write them as $\psi^{-1}\left(\psi\left(k_{t}\left(\psi^{-1}(z)\right)\right)\right)=$ $\psi^{-1}\left(\Psi_{t}(z)\right)$. The observation that

$$
\frac{\Psi_{t}(z)-1}{z-1}=\frac{e^{-t}(1+z)}{1+\Psi_{t}(z)}
$$

is also used. We wish to estimate $\left\|Q_{t}^{+}\right\|_{p}$. For the term $(1+z) /\left(1+\Psi_{t}(z)\right)$ we have

$$
\frac{1+z}{1+\Psi_{t}(z)}=\frac{e^{t / 2}(1+z)}{e^{t / 2}+\sqrt{z^{2}+e^{t}-1}} .
$$


An application of Schwarz's Lemma in $\pi^{+}$gives $\left|(1+z) /\left(e^{t / 2}+\sqrt{z^{2}+e^{t}-1}\right)\right| \leq$ 1 for all $z \in \pi^{+}$so we have

$$
\sup \left\{\left|(1+z) /\left(1+\Psi_{t}(z)\right)\right|^{2-2 / p}: z \in \pi^{+}\right\} \leq e^{(1-1 / p) t} .
$$

Thus from (3.14) we obtain $\left|Q_{t}^{+}(f)(z)\right| \leq e^{-t / p} \mid f\left(\Psi_{t}(z) \mid\right.$, and by integration,

$$
\left\|Q_{t}^{+}(f)\right\|_{p} \leq e^{-t / p}\left\|f \circ \Psi_{t}\right\|_{p}
$$

Next consider the composition operator $W_{t}(f)=f \circ \Psi_{t}$ on $H^{p}\left(\pi^{+}\right)$. Since $\Psi_{t}(z)=e^{-t / 2} \sqrt{z^{2}+e^{t}-1}$, using the notation of Lemma 1 we obtain $W_{t}=$ $C_{\left(e^{t}-1\right)} \circ M_{\left(e^{-t / 2}\right)}$. From Lemma 1 we have $\left\|C_{\left(e^{t}-1\right)}\right\|_{p}=1$ and $\left\|M_{\left(e^{-t / 2}\right)}\right\|_{p}=$ $e^{t / 2 p}$, so $\left\|W_{t}\right\|_{p} \leq e^{t / 2 p}$. From this and (3.15) the desired estimate follows.

Next we show that $\{z: \operatorname{Re} z \leq-1 / 2 p\} \subseteq \sigma\left(\mathscr{K}_{p}\right)$. Suppose $\lambda \in \mathbb{C}$ with $\operatorname{Re}(\lambda) \leq-1 / 2 p$. Choose $n$ integer such that $\operatorname{Re}(2 \lambda+n)>-1 / p$ and let

$$
P_{n, \lambda}(z)=\sum_{k=1}^{n-1}(-1)^{k} \frac{\lambda+1}{k}\left\{\left(\begin{array}{c}
n-1 \\
k
\end{array}\right)-\left(\begin{array}{c}
n-1 \\
k-1
\end{array}\right)\right\} z^{k}-(-1)^{n} \frac{\lambda+1}{n} z^{n}
$$

Then the function $\exp \left(P_{n, \lambda}(z)\right)(1-z)^{2 \lambda+n}$ is in $H^{p}$. If $\lambda-\mathscr{K}_{p}$ is invertible then the differential equation

$$
\lambda y(z)+z \frac{1-z}{1+z} y^{\prime}(z)+\frac{1-z}{1+z} y(z)=(\lambda+1) \exp \left(P_{n, \lambda}(z)\right)(1-z)^{2 \lambda+n}
$$

has an analytic solution $y(z)$ on $\mathbb{D}$ and $y(z) \in \operatorname{domain}\left(\mathscr{K}_{p}\right) \subset H^{p}$. A computation shows that the function $y(z)=\exp \left(P_{n, \lambda}(z)\right)(1-z)^{2 \lambda}$ is the only analytic solution of (3.16). But $y(z)$ is not in $H^{p}$ because $\operatorname{Re}(2 \lambda) \leq-1 / p$; thus $\lambda-\mathscr{K}_{p}$ is not invertible for $\operatorname{Re}(\lambda) \leq-1 / 2 p$.

Finally we conclude the proof. Using the spectral theorem [9, Theorem 9.4] from the general theory of strongly continuous semigroups we have

$$
\left\{z:|z| \leq e^{-t / 2 p}\right\} \subseteq\left\{e^{\lambda t}: \lambda \in \sigma\left(\mathscr{K}_{p}\right)\right\} \subseteq \sigma\left(Q_{t}\right),
$$

thus $\left\|Q_{t}\right\|_{p} \geq e^{-t / 2 p}$. It follows from the first part of the proof that this inequality and the set inclusions (3.17) all hold as equalities, giving parts (i) and (ii) of the theorem. For part (iii), choose the orbit $\gamma(t)=\left\{k_{t}(z): t \geq 0\right\}$ as a path of integration in the integral (3.4) and observe that the relation

$$
\frac{1+k_{t}(z)}{1-k_{t}(z)} \frac{\partial k_{t}(z)}{\partial t}=-k_{t}(z)
$$

holds for each $z$ and $t$. Thus we obtain $K_{p}$ as a Laplace transform

$$
K_{p}(f)(z)=\int_{0}^{\infty} \frac{k_{t}(z)}{z} f\left(k_{t}(z)\right) d t=\int_{0}^{\infty} Q_{t}(f)(z) d t .
$$

From this we obtain

$$
\left\|K_{p}\right\| \leq \int_{0}^{\infty}\left\|Q_{t}\right\|_{p} d t=\int_{0}^{\infty} e^{-t / 2 p} d t=2 p
$$

On the other hand,

$$
\sigma\left(K_{p}\right)=\left\{-1 / \lambda: \lambda \in \sigma\left(\mathscr{K}_{p}\right)\right\} \cup\{0\}=\{z:|z-2 p| \leq 2 p\} ;
$$

thus $\left\|K_{p}\right\| \geq 2 p$. This completes the proof. 
The adjoint semigroup. On the Hilbert space $H^{2}$ with the standard basis, the operator $K_{2}$ is represented by matrix

$$
K=\left(\begin{array}{l}
1 \\
2 / 2,1 / 2, \quad 0 \\
2 / 3,2 / 3,1 / 3, \\
\ldots \ldots \ldots \ldots \ldots
\end{array}\right)
$$

The matrix for the adjoint $K_{2}^{*}$ is the transpose of (3.18). A calculation shows that $K_{2}^{*}$ can be written as an integral in the form

$$
K_{2}^{*}(f)(z)=\frac{1}{z}(L(f)(z)-L(f)(0)),
$$

where $L$ is

$$
L(f)(z)=\frac{z+1}{z-1} \int_{1}^{z} f(\zeta) d \zeta
$$

It is interesting that the operator $L$ can also be obtained through a semigroup of weighted composition operators, which we discuss next in the $H^{p}$ setting. We denote by $L_{p}$ the operator $L$ on $H^{p}$.

Let $F(z)=1 /\left(1-z^{2}\right) \in \mathscr{P}$ and $G(z)=F(z)(1-z)^{2}=(1-z) /(1+z)$. According to [3, Theorem 3.3] there is a semigroup $\left\{\lambda_{t}(z): t \geq 0\right\}$ of analytic functions mapping $\mathbb{D}$ into itself with generator $G(z)$. The common DenjoyWolff point of $\lambda_{t}, t \geq 0$ is now the point $1 \in \partial \mathbb{D}$. Further it follows from [3, Theorem 3.7] that $\lambda_{t}(z)=\theta^{-1}(\theta(z)+t)$ where the function $\theta(z)$ is determined by $\theta^{\prime}(z)=1 / G(z), \theta(0)=0$. We find $\theta(z)=2 \log (1 /(1-z))-z$ so $\lambda_{t}(z)$ satisfies

$$
1-\lambda_{t}(z)=e^{-t / 2}(1-z) e^{\left(z-\lambda_{t}(z)\right) / 2}
$$

for each $t \geq 0$ and $z \in \mathbb{D}$. Since also $\lambda_{t}(z)=z+\int_{0}^{t} G\left(\lambda_{s}(z)\right) d s$ [3, Theorem 1.1] and $u(z)=\operatorname{Re}(G(z))>0$, we have $\operatorname{Re}\left(z-\lambda_{t}(z)\right) \leq 0$ and this together with (3.21) gives

$$
\left|\frac{1-\lambda_{t}(z)}{1-z}\right| \leq e^{-t / 2} \quad \text { for all } t \geq 0 \text { and } z \in \mathbb{D} .
$$

Next let $w=x+i y \in \mathbb{D}$ fixed. The orbit $O_{w}=\left\{\lambda_{t}(w): t \geq 0\right\}$ is the preimage of the half-line $\{\theta(w)+t: t \geq 0\}$ under $\theta(z)$, a function which is real on the real axis and univalent. Thus $O_{w}$ stays always on the same side of the real axis. Writing $G=u+i v, u>0$, and $v(z)=-2 \operatorname{Im}(z) /|1+z|^{2}$, we see that $v\left(\lambda_{s}(w)\right)$ does not change sign as $s$ moves from 0 to $\infty$. From these observations and the relation

$$
\left|1+\lambda_{t}(w)\right|^{2}=\left(1+x+\int_{0}^{t} u\left(\lambda_{s}(w)\right) d s\right)^{2}+\left(y+\int_{0}^{t} v\left(\lambda_{s}(w)\right) d s\right)^{2}
$$

we find that $\left|1+\lambda_{t}(w)\right|$ increases with $t$. Since $\lambda_{0}(w)=w$ and $w$ was arbitrary we conclude

$$
\left|\frac{1+z}{1+\lambda_{t}(z)}\right| \leq 1 \quad \text { for each } t \geq 0 \text { and } z \in \mathbb{D} .
$$

Next consider the operator semigroup $\left\{\Lambda_{t}: t \geq 0\right\}$ given on $H^{p}$ by

$$
\Lambda_{t}(f)(z)=\frac{\partial \lambda_{t}(z)}{\partial t} f\left(\lambda_{t}(z)\right)=\frac{1+z}{1+\lambda_{t}(z)} \frac{1-\lambda_{t}(z)}{1-z} f\left(\lambda_{t}(z)\right) .
$$


From [17, Theorem 1] $\left\{\Lambda_{t}\right\}$ is strongly continuous and has generator

$$
\mathscr{L}_{p}(f)(z)=\left(\frac{1-z}{1+z} f(z)\right)^{\prime} .
$$

Theorem 2. Suppose $p>1$. Then $R\left(0, \mathscr{L}_{p}\right)=L_{p},\left\|L_{p}\right\|=2 p /(p-1)$, and

$$
\sigma\left(L_{p}\right)=\{z:|z-p /(p-1)| \leq p /(p-1)\} .
$$

Proof. For each complex $\lambda$ with $\operatorname{Re} \lambda<-(p-1) / 2 p$ the function $q_{\lambda}(z)=$ $(1+z)(1-z)^{-(2 \lambda+1)} e^{-\lambda z}$ is in $H^{p}$ and is an eigenfunction of $\mathscr{L}_{p}$ with corresponding eigenvalue $\lambda$. Thus

$$
\{z: \operatorname{Re} z \leq-(p-1) / 2 p\} \subseteq \sigma\left(\mathscr{L}_{p}\right) .
$$

Next from (3.22) and (3.23) we have $\left|\Lambda_{t}(f)(z)\right| \leq e^{-t / 2}\left|f\left(\lambda_{t}(z)\right)\right|$; thus

$$
\left\|\Lambda_{t}(f)\right\|_{p} \leq e^{-t / 2}\left\|f \circ \lambda_{t}\right\|_{p} \leq e^{-t / 2}\left(\frac{1+\left|\lambda_{t}(0)\right|}{1-\left|\lambda_{t}(0)\right|}\right)^{1 / p}\|f\|_{p} .
$$

For the bound of the norm of $f \circ \lambda_{t}$ see for example [7, p. 29]. Now $\lambda_{t}(0)$ is a positive real less than 1 and from (3.21) we have $1-\lambda_{t}(0)=e^{-t / 2} e^{-\lambda_{t}(0) / 2}$. Thus

$$
\left\|\Lambda_{t}\right\|_{p} \leq e^{-t / 2}\left(1+\lambda_{t}(0)\right)^{1 / p} e^{t / 2 p} e^{\lambda_{t}(0) / 2 p} \leq(2 \sqrt{e})^{1 / p} e^{-t(p-1) / 2 p} .
$$

From the general theory of strongly continuous semigroups it follows that the resolvent set of $\mathscr{L}_{p}$ contains the set $\{z: \operatorname{Re} z>-(p-1) / 2 p\}$ and from (3.26) we have $\sigma\left(\mathscr{L}_{p}\right)=\{z: \operatorname{Re} z \leq-(p-1) / 2 p\}$. A computation gives $R\left(0, \mathscr{L}_{p}\right)=$ $L_{p}$, and $\sigma\left(L_{p}\right)=\left\{-1 / \lambda: \lambda \in \sigma\left(\mathscr{L}_{p}\right)\right\} \cup\{0\}=\{z:|z-p /(p-1)| \leq p /(p-1)\}$. Thus $\left\|L_{p}\right\| \geq 2 p /(p-1)$. On the other hand, from [15, Theorem 2] the operator

$$
A_{p}(f)(z)=\left(\frac{1}{z-1}\right) \int_{1}^{z} f(\zeta) d \zeta
$$

has norm equal to $p /(p-1)$. Since $L_{p}$ is $(1+z) A_{p}$ the conclusion about $\left\|L_{p}\right\|$ follows.

Remark 1. We have shown in the course of the above proof that $\left\|\Lambda_{t}\right\|_{p} \leq$ $(2 \sqrt{e})^{1 / p} e^{-t(p-1) / 2 p}$. On the other hand, $\left\|\Lambda_{t}\right\|_{p} \geq e^{-t(p-1) / 2 p}$ because $\sigma\left(\Lambda_{t}\right) \supseteq$ $\left\{e^{\lambda t}: \lambda \in \sigma\left(\mathscr{L}_{p}\right)\right\}=\left\{z:|z| \leq e^{-t(p-1) / 2 p}\right\}$. No doubt the correct values are $\left\|\Lambda_{t}\right\|_{p}=e^{-t(p-1) / 2 p}$ and $\sigma\left(\Lambda_{t}\right)=\left\{z:|z| \leq e^{-t(p-1) / 2 p}\right\}$ and would both follow from the correct value of the norm, $\left\|C_{t}\right\|_{p}=e^{t / 2 p}$, of the composition operator $C_{t}(f)=f \circ \lambda_{t}$. We were not able to obtain the above value for the norm of $C_{t}$, but a strong evidence in favor is that $\lambda_{t}(z)$ is univalent in $\mathbb{D}$ with DenjoyWolff point 1 and no other fixed point on $\partial \mathbb{D}$, and its angular derivative at 1 is $\lambda_{t}^{\prime}(1)=e^{-t / 2}$ (the value of $\lambda_{t}^{\prime}(1)$ is obtained easily from (3.21)). The norm of $C_{t}$ is thus expected to be $e^{t / 2 p}=\left(1 / \lambda_{t}^{\prime}(1)\right)^{1 / p}$.

\section{THE OPERATORS $T_{F}$}

We now return to the operators $T_{F}$. The following theorem gives an upper bound for the norm. 
Theorem 3. Suppose $1 \leq p<\infty$ and $F \in \mathscr{P}$. Then the operator $T_{F}$ is bounded on $H^{p}$ and $\left\|T_{F}\right\|_{p} \leq 2 p \operatorname{Re}(1 / F(0))+|\operatorname{Im}(1 / F(0))|$.

Proof. Let $\mu$ be the measure in the Herglotz representation of $1 / F(z)$ and $\gamma=\operatorname{Im}(1 / F(0))$. For each $f \in H^{p}$ we have

$$
\begin{aligned}
T_{F}(f)(z) & =\frac{1}{z} \int_{0}^{z} f(\xi)\left(\int_{\partial \mathbb{D}} \frac{\zeta+\xi}{\zeta-\xi} d \mu(\zeta)+i \gamma\right) d \xi \\
& =\int_{\partial \mathbb{D}}\left(\frac{1}{z} \int_{0}^{z} f(\xi) \frac{\zeta+\xi}{\zeta-\xi} d \xi\right) d \mu(\zeta)+i \gamma \frac{1}{z} \int_{0}^{z} f(\xi) d \xi \\
& =\int_{\partial \mathbb{D}} K_{p}^{\zeta}(f)(z) d \mu(\zeta)+i \gamma J(f)(z),
\end{aligned}
$$

where $K_{p}^{\zeta}$ is a rotated version of the operator $K_{p}$ and $J$ is the operator of integration. Clearly $\left\|K_{p}^{\zeta}\right\|=\left\|K_{p}\right\|=2 p$ and $\|J\|_{p}=1$. From (4.1) we have $T_{F}=\int_{\partial \mathbb{D}} K_{p}^{\zeta} d \mu+i \gamma J$, so

$$
\begin{aligned}
\left\|T_{F}\right\|_{p} & \leq \int_{\partial \mathbb{D}}\left\|K_{p}^{\zeta}\right\| d \mu(\theta)+|\gamma|=2 p \mu(\partial \mathbb{D})+|\gamma| \\
& =2 p \operatorname{Re}(1 / F(0))+|\operatorname{Im}(1 / F(0))|,
\end{aligned}
$$

completing the proof.

Corollary 1. Suppose $1 \leq p<\infty$. For all semigroups of the form (2.7) on $H^{p}$ the point $\lambda=0$ is in the resolvent set of the infinitesimal generator $\mathscr{B}_{p}$, and $R\left(0, \mathscr{B}_{p}\right)=T_{F}$.

Remark 2. Under the additional condition $F(0)=1$ for functions in $\mathscr{P}$ one has $\left\|T_{F}\right\|_{p} \leq 2 p$ with equality if $F(z)=(1-z) /(1+z)$ or any of its rotations $F(\zeta z)$. We do not know if there are other $F$ with $F(0)=1$ for which $\left\|T_{F}\right\|_{p}=$ $2 p$. If $\operatorname{Re} F(0)=0$, in which case $F$ is an imaginary constant, the maximum norm permitted by the bound in Theorem 3 is also realized.

Elementary initial considerations reveal that the compactness of $T_{F}$ is closely related to the geometric behavior of the range of $F(z)$ near $z=0$. If $F(z)$ is bounded away from zero then $1 / F \in H^{\infty}$ and $T_{F}$ is compact. Less obvious is the fact that if the range of $F(z)$ in a neighborhood of zero is contained in an angle with vertex $z=0$ and opening less than $\pi$ then $T_{F}$ is compact. This follows from a calculation similar to the one in the proof of [16, Theorem 2]. Thus for $T_{F}$ to be noncompact it is necessary that in each neighborhood of zero, no angle with vertex $z=0$ and opening less than $\pi$ contain the portion of the range in this neighborhood. Examples of such $F$ are $F(z)=1-z$ or $F(z)=(1-z) /(1+z)$ inducing respectively the operators $\mathscr{C}$ and $K_{p}$. We will come back to this geometric relation, expressing it in Remark 3 in terms of an angular derivative.

Theorem 4. Suppose $1 \leq p<\infty$. Let $F(z) \in \mathscr{P}$ and $\mu$ the measure corresponding to $1 / F(z)$. The following conditions are equivalent:

(i) $T_{F}$ is a compact operator on $H^{p}$.

(ii) $\mu$ has no point masses on $\partial \mathbb{D}$.

Proof. If $F(z)=i \gamma$ is an imaginary constant then $T_{F}$ is compact and the corresponding measure is the zero measure. We may therefore assume that $\operatorname{Re} F(0)>0$. 
Let $\mid\left\{\varphi_{t}\right\}$ be the semigroup with generator $-z F(z)$. The operator semigroups $T_{t}(f)(z)=f\left(\varphi_{t}(z)\right)$ and $S_{t}(f)(z)=\left(\varphi_{t}(z) / z\right) f\left(\varphi_{t}(z)\right)$ have generators $\mathscr{A}_{p}$ and $\mathscr{B}_{p}$ given by (2.6) and (2.8) respectively, and satisfy $\left\|T_{t}\right\|_{p} \leq 1$ and $\left\|S_{t}\right\|_{p} \leq 1$. Thus the right half-plane $\{z: \operatorname{Re} z>0\}$ is contained in the resolvent sets of both $\mathscr{A}_{p}$ and $\mathscr{B}_{p}$ so in particular $R\left(\lambda_{0}, \mathscr{A}_{p}\right)$ and $R\left(\lambda_{0}, \mathscr{B}_{p}\right)$, $\lambda_{0}=F(0)$, are both bounded. Further from Corollary 1 and the resolvent equation [9, Lemma 1.3], $T_{F}$ is compact if and only if $R\left(\lambda_{0}, \mathscr{B}_{p}\right)$ is compact. Let $M_{z}: H^{p} \rightarrow H^{p}$ be the operator of multiplication by $z, M_{z}(f)(z)=z f(z)$. Evidently $M_{z} \circ S_{t}=T_{t} \circ M_{z}$ for $t \geq 0$. An easy computation shows that this intertwining relation is inherited by the infinitesimal generators $\mathscr{A}_{p}$ and $\mathscr{B}_{p}$, and by the corresponding resolvent functions on the common part of the resolvent sets. More precisely at $\lambda_{0}$ from a computation we have

$$
R\left(\lambda_{0}, \mathscr{A}_{p}\right)(f)(z)=\frac{1}{\lambda_{0} h(z)} \int_{0}^{z} f(\zeta) h^{\prime}(\zeta) d \zeta
$$

where $h(z)$ is the associated univalent function given by (2.2). A similar computation gives

$$
R\left(\lambda_{0}, \mathscr{B}_{p}\right)(f)(z)=\frac{1}{\lambda_{0} z h(z)} \int_{0}^{z} \zeta f(\zeta) h^{\prime}(\zeta) d \zeta
$$

Clearly,

$$
M_{z} \circ R\left(\lambda_{0}, \mathscr{B}_{p}\right)=R\left(\lambda_{0}, \mathscr{A}_{p}\right) \circ M_{z} .
$$

It follows from (4.6) that $R\left(\lambda_{0}, \mathscr{B}_{p}\right)$ and $R\left(\lambda_{0}, \mathscr{A}_{p}\right)$ are simultaneously compact. Further from [1, Theorem 3.1] $R\left(\lambda_{0}, \mathscr{A}_{p}\right)$ is compact if and only if the associated univalent function $h(z)$ is in $H^{p}$ for all finite $p$. Finally from [3, Theorem 4.10] (with simple modification in its proof to include the case when $F(0)$ is not real) this is equivalent to $\mu\{w\}=0$ for each $w \in \partial \mathbb{D}$ for the measure $\mu$ representing $1 / F(z)$. This finishes the proof.

Remark 3. If $\mu$ is the measure in the Herglotz representation of $1 / F(z)$ and $w \in \partial \mathbb{D}$ then

$$
\begin{aligned}
\frac{1}{F(z)} & =\int_{\partial \mathbb{D}} \frac{\zeta+z}{\zeta-z} d \mu(\zeta)+i \operatorname{Im}\left(\frac{1}{F(0)}\right) \\
& =\mu(w) \frac{w+z}{w-z}+\int_{\partial \mathbb{D}} \frac{\zeta+z}{\zeta-z} d \mu_{1}(\zeta)+i \operatorname{Im}\left(\frac{1}{F(0)}\right)
\end{aligned}
$$

where $0 \leq \mu(w)<\infty$ is the point mass at $w$ and $\mu_{1}$ is the rest of $\mu$. Then

$$
\frac{w-z}{F(z)}=\mu(w)(w+z)+\int_{\partial \mathbb{D}}(\zeta+z) \frac{w-z}{\zeta-z} d \mu_{1}(\zeta)+(w-z) i \operatorname{Im}\left(\frac{1}{F(0)}\right) .
$$

Now let $z \rightarrow w$ nontangentially. Since $|(w-z) /(\zeta-z)|$ remains bounded and $\mu_{1}$ is a finite measure the limiting value of the integral is zero. Thus $\lim ((w-z) / F(z))=2 w \mu(w)$ and with the interpretation $1 / 0=\infty$,

$$
\lim \frac{F(z)}{z-w}=-\frac{\bar{w}}{2 \mu(w)} \quad(z \rightarrow w \text { nontangentially }) .
$$

Clearly, for the limit in (4.7) to be finite it is necessary that the nontangential limit $F(w)=\lim F(z)$ has the value zero, that is the range of $F(z)$ "touches" the imaginary axis at 0 . Interpreting $F(z) /(z-w)$ as a quotient of differences 
and its limiting value $F^{\prime}(w)$ as the derivative at $w$ we have from (4.7) that $F^{\prime}(w)$ is never zero and it is finite if and only if $\mu(w)>0 . F^{\prime}(w)$ is called the angular derivative of $F(z)$ at $w$ (see [4, §298] or [13] for details). Its finiteness means that $F(z)$ is conformal at $w$. In particular if $F^{\prime}(w)$ is finite the range $F(\mathbb{D})$ touches the imaginary axis at 0 in an essential way. According to Theorem 4 this is a situation that prohibits compactness of $T_{F}$. Concluding, $T_{F}$ is compact if and only if at each $w \in \partial \mathbb{D}$ at which the nontangential limit $\lim _{z \rightarrow w} F(z)$ is zero, $F(z)$ has no finite angular derivative. This phenomenon of the close relation between angular derivative and compactness was studied extensively for composition operators in [12 and 14].

\section{The Cesàro operator}

The exact value of $\|\mathscr{C}\|_{p}$ for $1 \leq p<2$ remains unknown. We show here that $\|\mathscr{C}\|_{p}>p$ for $p$ sufficiently close to 1 so, in spite of expectation, $\|\mathscr{C}\|_{p}=p$ does not hold for $1 \leq p<2$ as it does for $p \geq 2$.

Proposition 1. (i) There is a $p_{0}>1$ such that $\|\mathscr{C}\|_{p}>p$ for $1 \leq p<p_{0}$.

(ii) For each $p \in[1,2) \quad\|\mathscr{C}\|_{p} \leq p+1 / 2$.

Proof. (i) Let $g(z)=\mathscr{C}(1)(z)=(1 / z) \log (1 /(1-z))$. Since $g(z)$ has no zero in $\mathbb{D}, \log |g(z)|$ is harmonic and satisfies the mean value property,

$$
\log |g(0)|=\frac{1}{2 \pi} \int_{0}^{2 \pi} \log \left|g\left(r e^{i \theta}\right)\right| d \theta,
$$

for each $r<1$. Applying the arithmetic-geometric mean inequality on (5.1) we obtain

$$
1=|g(0)|=\exp \left(\frac{1}{2 \pi} \int_{0}^{2 \pi} \log \left|g\left(r e^{i \theta}\right)\right| d \theta\right)<\frac{1}{2 \pi} \int_{0}^{2 \pi}\left|g\left(r e^{i \theta}\right)\right| d \theta \leq\|g\|_{1} .
$$

Set $p_{0}=\|g\|_{1}$. Since $\|1\|_{p}=1$ for each $p$ and $\|g\|_{p}$ is increasing in $p$ we have for $1 \leq p<p_{0}$

$$
\|\mathscr{C}\|_{p} \geq\|\mathscr{C}(1)\|_{p}=\|g\|_{p}>\|g\|_{1}=p_{0}>p
$$

proving (i). For (ii), the identity $1 /(1-z)=1 / 2(1+(1+z) /(1-z))$ gives $\mathscr{C}=$ $1 / 2\left(K_{p}+J\right)$ where $K_{p}$ is the operator (3.4) and $J$ the operator of integration. Since $\|J\|_{p}=1$ and $\left\|K_{p}\right\|=2 p$ we have $\|\mathscr{C}\|_{p} \leq p+1 / 2$.

Remark 4. The value of $p_{0}$ in Proposition 1 is

$$
p_{0}=\|g\|_{1}=\frac{2}{\pi} \int_{0}^{\pi / 2}\left(\log ^{2}(2 \cos \theta)+\theta^{2}\right)^{1 / 2} d \theta .
$$

A numerical computation (done with help from Professor W. Julian) has given $p_{0}=1.10$ correct to two decimal places. In summary the following is known about $\|\mathscr{C}\|_{p}$ :

(i) $\|\mathscr{C}\|_{p}=p$ for $p \geq 2$.

(ii) $p_{0}<\|\mathscr{C}\|_{p} \leq p+1 / 2$ for $1 \leq p \leq p_{0}$.

(iii) $p \leq\|\mathscr{C}\|_{p} \leq p+1 / 2$ for $p_{0}<p<3 / 2$.

(iv) $p \leq\|\mathscr{C}\|_{p}<2$ for $3 / 2 \leq p<2$. 


\section{FINAL QUESTIONS AND REMARKS}

(i) The operator $K_{p}$ and its rotated versions $K_{p}^{\zeta}, \zeta \in \partial \mathbb{D}$, are of norm $2 p$ and as Theorem 4 suggests they are as noncompact as possible. Since the space $\mathscr{I}$ of compact operators is closed, the distance $\left\|K_{p}^{\zeta}\right\|_{e}=\inf \left\{\left\|K_{p}^{\zeta}-T\right\|: T \in \mathscr{I}\right\}$ of $K_{p}^{\zeta}$ from $\mathscr{I}$ is positive. $\left\|K_{p}^{\zeta}\right\|_{e}$ is called the essential norm of $K_{p}^{\zeta}$. Is it true that $\left\|K_{p}^{\zeta}\right\|_{e}=2 p$ ? More generally if the measure $\mu$ corresponding to $1 / F$ has decomposition $\mu=\sum c_{i} \delta_{w_{i}}+\mu_{c}$, where $\delta_{w_{i}}$ is the unit Dirac mass at $w_{i} \in \partial \mathbb{D}$ and $\mu_{c}$ is the continuous part of $\mu$, is it true that $\left\|T_{F}\right\|_{e}=2 p\left(\sum c_{i}\right)$ ?

(ii) On the Hilbert space $H^{2}$ it is easy to see that $T_{F}$ is a normal operator (: commutes with its adjoint) if and only if $F=c$ is a constant function. An operator on a Hilbert space is subnormal if it is the restriction of a normal operator on an invariant subspace. The Cesàro operator has been studied extensively on the Hilbert space $H^{2}$ (see $[5,11]$ and the references therein) proving that $\mathscr{C}$ is subnormal. The proof of subnormality of $\mathscr{C}$ in [5] is essentially based on the fact that $\mathscr{C}$ is the resolvent operator of a semigroup $\left\{S_{t}\right\}$ of the form (2.7). Recall that $\mathscr{C}=T_{1-z}$. Which other choices of $F(z) \in \mathscr{P}$ give subnormal $T_{F}$ ? Since very nonzero subnormal compact operator is normal, it follows from Remark 3 that if $T_{F}$ is subnormal and not normal then $F$ must have 0 as a boundary value at some $w \in \partial \mathbb{D}$ and have nonzero angular derivative at $w$. This is satisfied for functions of the form $F(z)=(1-z) g(z)$ (taking $w=1)$ where $g$ is analytic in a neighborhood of $\mathbb{D} \cup\{1\}$ and $g(1) \neq 0$. An especially simple family of such $F$ is $F(z)=(1-z)(1+s z), 0 \leq s \leq 1$. The semigroups corresponding to these $F$ are not simple enough, except when $s=0$, to provide information on subnormality of $T_{F}$.

\section{REFERENCES}

1. A. Aleman, Compactness of resolvent operators generated by a class of composition semigroups on $H^{p}$, J. Math. Anal. Appl. 147 (1990), 171-179.

2. _. Personal communication, 1990.

3. E. Berkson and H. Porta, Semigroups of analytic functions and composition operators, Michigan Math. J. 25 (1978), 101-115.

4. C. Carathéodory, Theory of functions of a complex variable. II, Chelsea, New York, 1954.

5. C. C. Cowen, Subnormality of the Cesàro operator and a semigroup of composition operators, Indiana Univ. Math. J. 33 (1984), 305-318.

6. J. A. Deddens, Analytic Toeplitz and composition operators, Canad. J. Math. 24 (1972), 859-865.

7. P. L. Duren, Theory of $H^{p}$ spaces, Pure and Appl. Math., vol. 38, Academic Press, New York and London, 1970.

8. _ Univalent functions, Springer-Verlag, New York and Berlin, 1983.

9. J. A. Goldstein, Semigroups of linear operators and applications, Oxford Univ. Press, London and New York, 1985

10. K. Hoffman, Banach spaces of analytic functions, Prentice-Hall, Englewood Cliffs, N.J., 1962.

11. T. L. Kriete and D. Trutt, The Cesàro operator on $l^{2}$ is subnormal, Amer. J. Math. 93 (1971), 215-225.

12. B. D. MacCluer and J. H. Shapiro, Angular derivatives and compact composition operators on the Hardy and Bergmann spaces, Canad. J. Math. 38 (1986), 878-906.

13. Ch. Pommerenke, On the angular derivative and univalence, Anal. Math. 3 (1977), 291-297. 
14. J. H. Shapiro, The essential norm of a composition operator, Ann. of Math. (2) 125 (1987), 375-404.

15. A. G. Siskakis, Composition semigroups and the Cesàro operator on $H^{p}, \mathrm{~J}$. London Math. Soc. (2) 36 (1987), 153-164.

16. _ $\ldots$, On a class of composition semigroups in Hardy spaces, J. Math. Anal. Appl. 127 (1987), 122-129.

17. __ Weighted composition semigroups on Hardy spaces, Linear Algebra Appl. 84 (1986), 359-371.

Department of Mathematics, University of Thessaloniki, 54006 Thessaloniki, Greece E-mail address: cbcz02@grtheun1.bitnet 